\title{
Dynamic Orientation of Nuclei at Low Temperatures
}

\author{
W. de Boer* \\ CERN, Geneva, Switzerland
}

(Received June 2, 1975)

A study has been made of the mechanisms of dynamic polarization of nuclei in organic materials at temperatures below $1 \mathrm{~K}$ and in magnetic fields up to $50 \mathrm{kG}$. After a summary of the mechanisms of dynamic polarization, the experimental results on 1,2-propanediol, doped with paramagnetic $\mathrm{Cr}^{\mathrm{V}}$ complexes, are compared quantitatively with a spin temperature model of Borghini, in which it is assumed that the nuclear polarization is obtained via thermal contact with a dynamically cooled electron spin-spin interaction reservoir. The strong influence of this latter reservoir was apparent from the transient behavior of different nuclear spin systems contained in the same sample. The abovementioned spin temperature model has the unique property of not using the simplifying "high-temperature approximation" and it was the first time that this model has been found to be in reasonable agreement with experimental data in the $100 \mathrm{mK}$ region. Furthermore, dynamic polarization experiments with the free radical $B D P A$ are reviewed. These measurements show clearly the contributions of different mechanisms of dynamic polarization. The results are found to be in agreement with formulas derived from the spin temperature theory after introduction of some simplifying assumptions in order to be able to deal with low temperatures.

\section{INTRODUCTION}

Dynamic polarization by electron-nucleus dipolar coupling has been studied for many years now. The interest in these experiments has come from two sides : first from the point of view of mechanisms of dynamic polarization itself, ${ }^{1-9}$ and second from nuclear and high-energy physics, where dynamic polarization is used for the production of polarized targets. ${ }^{10}$

\footnotetext{
*Present address: Randall Laboratorium of Physics, University of Michigan, Ann Arbor, Michigan.
} 
This study will be concerned with the mechanisms of dynamic polarization in organic materials under conditions which are suitable for obtaining high spin orientations, i.e., at low temperatures and in high magnetic fields. These materials can be doped with paramagnetic impurities, of which the spin of the "free" electron can be oriented easily in a magnetic field due to its strong magnetic moment. By microwave irradiation of the spin system it is possible to transmit the high degree of orientation of the electron spins to the nuclear spins, thus increasing the nuclear polarization.

Several schemes, effects, or mechanisms of dynamic polarization have been proposed and most of them were experimentally verified. ${ }^{4-9,11-18}$ In the case of a solid it is in general not easy to find out which mechanism is responsible for dynamic polarization, because several mechanisms may act simultaneously. The situation is especially complicated by the magnetic interactions between similar spins, which may be appreciable in a solid. In the early theories of dynamic polarization in solids, these spin-spin interactions were normally neglected or only partially taken into account (see the work by Overhauser, ${ }^{12}$ Bloembergen and Sorokin, ${ }^{17}$ Abragam and Proctor, ${ }^{18}$ and Jeffries ${ }^{2}$ ). However, later the important role of the electron spin-spin interactions was demonstrated in several dynamic polarization experiments (see the work by Borghini, ${ }^{4}$ Buishvili et al.,${ }^{5}$ Atsarkin and Rodak, ${ }^{6}$ and Wenckebach et $a l^{7}$ ). In the spin temperature theory, which forms the basic framework of the theory of magnetic resonance, these spin-spin interactions are taken into account, following Provotorov, by introducing a separate energy reservoir ${ }^{19,20}$ possessing its own temperature, which can be different from the temperature of the "Zeeman reservoir" under suitable conditions. In the following, the concept of a spin-spin interaction reservoir will appear to be invaluable for the interpretation of some results obtained at low temperatures. ${ }^{21-25} \mathrm{Up}$ until now, an exact comparison of these data with theory was difficult, because only few electron spin resonance data were available at low temperatures. Furthermore, a difficulty arises from the fact that the so-called high-temperature approximation in the spin temperature theory cannot be used at low temperatures. The extension of the spin temperature theory toward lower temperatures has been done only by taking second- or thirdorder terms into account ${ }^{26,27}$ or by neglecting the complicated dipolar interactions in cases where the spin system is mainly inhomogeneously broadened. ${ }^{28}$

In this paper we present electron spin resonance (ESR) data on two electron spin systems for which results on dynamic polarization experiments at low temperatures are available, namely diols doped with paramagnetic $\mathrm{Cr}^{\mathrm{V}}$ complexes $^{23,24}$ and toluenes doped with 1,2-bis-diphenylene-1-phenylallyl (BDPA), a free radical. ${ }^{25}$ The ESR data are then used to compare these dynamic polarization results with the spin temperature theory. 


\section{THEORY}

\subsection{Introduction}

The polarization $P$ of a system of spins $I$ is defined as

$$
P=\left\langle I_{z}\right\rangle / I
$$

which reduces in case of $I=\frac{1}{2}$ to

$$
P=n_{+}-n_{-}
$$

where $n_{+}$is the fraction of nuclei in the magnetic substate with $m=\left\langle I_{z}\right\rangle=$ $+\frac{1}{2}$ and $n_{-}$is the fraction with $m=-\frac{1}{2}$. If the spin system is in internal equilibrium, it can be characterized by a spin temperature $T_{S}$. The relative distribution of the spins over the various magnetic substates is then given by the Boltzmann factor

$$
n_{-} / n_{+}=\exp \left[\left(E_{+}-E_{-}\right) / k T_{S}\right]
$$

Here $k$ is the Boltzmann constant and $E_{ \pm}= \pm \mu H$ is the energy of the magnetic substates with populations $n_{ \pm}$, respectively; $H$ is the external magnetic field; and $\mu$ is the magnetic moment of the nuclei under consideration. The polarization of a spin system with $I=\frac{1}{2}$ is related to the spin temperature $T_{S}$ by

$$
P=\tanh \left(\mu H / k T_{S}\right)
$$

as follows from Eqs. (2) and (3). Equation (4) is a special case of the more general Brillouin formula. At sufficiently high temperatures, where $\mu H \ll k T_{S}$, Eq. (4) can be approximated by

$$
P=\mu H / k T_{S}=\frac{1}{2} h v / k T_{S}
$$

where $h$ is Planck's constant and $v$ is the Larmor frequency of the spin system.

The first dynamic polarization scheme involving microwave-induced transitions between electron and nuclear spins was invented by Overhauser for the polarization of nuclei in metals $;{ }^{12}$ it was experimentally verified by Carver and Slichter. ${ }^{29}$ Later it was extended to the cases of strong hyperfine ${ }^{15,30,31}$ and dipolar interactions ${ }^{17,18,32-34}$ as usually found in liquids and solids. In the following we will consider only the case of a dipolar coupling between electron spins and nuclear spins in solids and discuss the different mechanisms which may give rise to dynamic polarization under such conditions. We will first consider the case in which the electron spin resonance absorption line is narrow compared with the nuclear Larmor frequency. We then consider the opposite case. In the first case the so-called solid-state effect will be the dominant polarization mechanism, while in the second case 
several mechanisms are possible, namely the differential solid-state effect, ${ }^{5,35}$ the cross-effect, ${ }^{36}$ or "dynamic polarization by cooling of the electron spinspin interaction reservoir." ${ }^{4,5}$ Special cases of practical interest will be calculated in more detail, and they will be compared with experimental results.

\subsection{Mechanisms of Dynamic Polarization}

We first consider briefly the simple case of an electron and a nuclear spin in a magnetic field; their Larmor frequencies are denoted by $v_{e}$ and $v_{n}$, respectively. As a consequence of the dipolar coupling between the spins, a microwave field has a certain probability to induce, besides the allowed transitions at a frequency $v_{e}$ (involving a single electron spin flip), the socalled forbidden transitions, which involve a simultaneous spin flip of the electron and proton spin. The forbidden transitions occur at frequencies $v_{e} \pm v_{n}$ for reasons of energy conservation. It is just the possibility of these forbidden transitions that may cause dynamic polarization, because of the large ratio between the nuclear and electron spin lattice relaxation times. ${ }^{1,2}$ In that case the electron returns to its thermal equilibrium state after a microwave-induced transition more quickly than does a nuclear spin. The electron spin is then ready to flip the next nuclear spin in the same direction, if there are many nuclei surrounding one electron spin. Spin diffusion (flipflop transitions between nuclear spins ${ }^{37}$ ) tends to maintain thermal equilibrium within the nuclear spin system, so the spins far away from the electron spin become polarized as well. In the following we will assume that spin diffusion is fast enough to maintain a homogeneous nuclear polarization, as is usually the case.

The above-mentioned mechanism has been called the solid-state effect; it was first observed by Abragam and Proctor for the two nuclear spin systems in LiF. ${ }^{18}$ It is called the resolved solid-state effect if the forbidden transitions do not overlap with the allowed transitions. The polarization versus irradiation frequency then shows two peaks of an opposite sign at the frequencies of the forbidden transitions. The transient behavior and steady-state polarization can be obtained from rate equations. ${ }^{2}$ We will not write these rate equations now, but consider first the complications which arise if the forbidden transitions are not resolved from the allowed transitions.

In the case of completely inhomogeneous broadening, which can be caused, for example, by hyperfine interactions or $g$-factor anisotropy, one can think of the resonance absorption line as consisting of many independent spin packets with a slightly different Larmor frequency. ${ }^{38}$ Application of microwave power saturates the forbidden transitions of two spin packets simultaneously if the electron spin resonance line is broader than the nuclear 
Larmor frequency; the net polarization will then be proportional to the relative difference of the intensities of the two packets, which cause opposite contributions to the polarization. ${ }^{5,35}$ Therefore the net polarization always will be reduced. This mechanism is called the differential solid effect.

The assumption of independent spin packets is seldom justified in solids with high spin concentrations, because of the spin--spin interactions between the electron spins. The spin temperature theory, ${ }^{3,19}$. which correctly takes into account the existence of the spin-spin interactions, may then be the proper approach to the problem, at least if the spin-spin interactions are strong enough to maintain thermal equilibrium within the different parts of the spin system. For homogeneous spin systems this equilibrium is always obtained, while for inhomogeneous spin systems, thermal equilibrium may still be obtained if cross-relaxation (flip-flop transitions between the electron spins) is sufficiently fast compared with spin lattice relaxation. ${ }^{4,6}$ For intermediate cases of inhomogeneous lines with slow cross-relaxation there exists no adequate theory. A phenomenological model for such circumstances has been given in the literature under the name of the cross-effect. ${ }^{36}$ The crosseffect might not be the complete explanation of the phenomena. Furthermore, an experimental verification is difficult because of the adjustable parameters in this model.

Fortunately, at low temperatures the electron spin lattice relaxation times are rather long, so that cross-relaxation is normally fast enough to maintain thermal equilibrium within a spin system. Therefore we will focus our attention on the spin temperature theory, which is described hereafter insofar as it is important for the theory of dynamic polarization.

We first consider a single spin system with dipolar interactions in an external magnetic field. Due to the spin-spin interactions, the Zeeman levels then have a certain width of the order of the local internal magnetic fields $H_{L}$. The spin temperature theory is based on the segregation of the relatively weak spin-spin interactions into a separate reservoir ${ }^{19,20}$ which possesses its own temperature $T_{S S}$. This temperature may be different from the temperature of the Zeeman reservoir, denoted by $T_{\mathrm{Z}}$. Then the spin system can be characterized by two temperatures: one for the Boltzmann distribution between the Zeeman levels, with a temperature $T_{Z}$, and a second one $\left(T_{S S}\right)$ describing the ordering of the spins in the local fields. A pictorial illustration is given in Fig. 1.

If the spin system is in thermal equilibrium with the lattice, the two temperatures are equal. However, under some experimental conditions they are different. ${ }^{3,6,19}$ For example, $T_{S S}$ can be changed by slightly offresonance irradiation of the spin system; it may become positive or negative, depending on the sign of $\Delta=v_{e}-v$, where $v$ is the irradiation frequency (see Fig. 1). Under such conditions Provotorov derived the evolution of the 


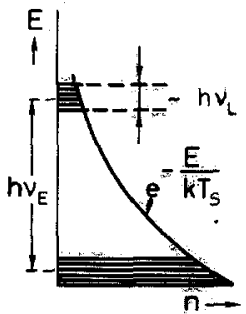

(a)

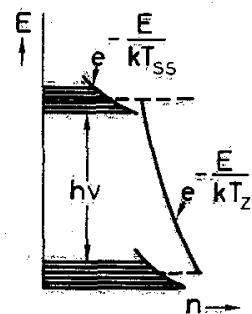

(b)

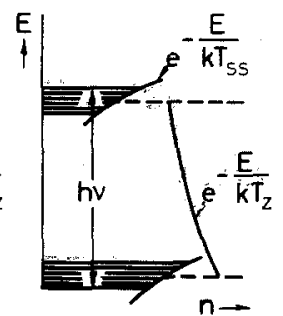

(c)

Fig. 1. (a) The distribution of the populations $n$ over the energy levels. In thermal equilibrium with the lattice this can be described by one spin temperature $T_{s}$ (b) During if irradiation at a frequency $v$, the spin system may be described by two spin temperatures, $T_{S S}$ and $T_{Z}$. If $v<v_{e}$ the populations become such that $T_{S S}<T_{Z}$, but $T_{S S}>0$. (c) As in (b) but for $y>y_{e}$; then $T_{S S}$ may become negative.

inverse spin temperatures of the Zeeman and spin-spin interaction reservoirs, denoted by $\alpha=h / k T_{\mathrm{Z}}$ and $\gamma=h / k T_{S S}$, respectively. ${ }^{19,20}$

In Provotorov's derivation, the so-called high-temperature approximation was used ; it consists in taking only the linear terms in the series expansion of the density matrix, which is only allowed at sufficiently small values of $\alpha v_{e}$ and $\gamma v_{L}$; the "local" internal frequency $v_{L}$, corresponding to $H_{L}$, will be defined more precisely later.

If there are nuclei surrounding the electron spins, the situation becomes more difficult, because the density matrix $\rho$ now involves three spin temperatures in general : $:^{4,5}$

$$
\rho=(1 / \Sigma) \exp \left(-\alpha v_{e} S_{z}-\beta v_{n} I_{z}-\gamma H_{S S}^{0}\right)
$$

Here $\Sigma$ is a normalization constant, $\beta$ is the inverse spin temperature of the nuclei, and $H_{S S}^{0}$ is the Hamiltonian for the secular part of the dipolar interactions between the electron spins, which is the part commuting with $S_{z}$. The dipolar interactions between electrons and nuclei and between the nuclei themselves are neglected.

Summing up the contributions of spin lattice relaxation, and the microwave-induced ${ }^{19}$ and cross-relaxation transitions ${ }^{20}$ of the electron spins, including the forbidden transitions $s^{8,39,40}$ in which a nuclear spin flips simultaneously, we obtain the following rate equations for spin one-half and using the high-temperature approximation; $; * 6$

$$
\begin{aligned}
\dot{P}_{e}= & =W_{0}\left(P_{e}+\frac{1}{2} \gamma \Delta\right)-W_{e}^{+}\left[P_{e}-P_{n}+\frac{1}{2} \gamma\left(\Delta+v_{n}\right)\right] \\
& -W_{e}^{-}\left[P_{e}+P_{n}+\frac{1}{2} \gamma\left(\Delta-v_{n}\right)\right]-\left(1 / T_{1 e}\right)\left(P_{e}-P_{0}\right)
\end{aligned}
$$




$$
\begin{aligned}
\dot{P}_{n}= & -W_{n}^{+}\left[P_{n}-P_{e}-\frac{1}{2} \gamma\left(\Delta+v_{n}\right)\right]-W_{n}^{-}\left[P_{n}+P_{e}+\frac{1}{2} \gamma\left(\Delta-v_{n}\right)\right] \\
& -\left(1 / \tau_{n S}\right)\left(P_{n}-\frac{1}{2} \gamma v_{n}\right)-\left[\left(P_{n}-P_{n 0}\right) / T_{1 n}\right] \\
\frac{1}{2} v_{L}^{2} \dot{\gamma}= & -W_{0} \Delta\left(P_{e}+\frac{1}{2} \gamma \Delta\right)-W_{e}^{+}\left(\Delta+v_{n}\right)\left[P_{e}-P_{n}+\frac{1}{2} \gamma\left(\Delta+v_{n}\right)\right] \\
& -W_{e}^{-}\left(\Delta-v_{n}\right)\left[P_{e}+P_{n}+\frac{1}{2} \gamma\left(\Delta-v_{n}\right)\right] \\
& +\left(v_{L}^{2} / v_{n}\right)\left(1 / \tau_{S n}\right)\left(P_{n}-\frac{1}{2} \gamma v_{n}\right)-\frac{1}{2} v_{L}^{2}\left[\left(\gamma-\beta_{L}\right) / T_{1 S s}\right]
\end{aligned}
$$

In the high-temperature approximation the electron and nuclear polarizations, denoted by $P_{e}$ and $P_{n}$, are related to $\alpha$ and $\beta$ by $P_{e}=-\frac{1}{2} \alpha v_{e}$ and $P_{n}=\frac{1}{2} \beta v_{n}$ in the case of spin one-half, where $P_{n}$ has the same sign as $\beta$ for a positive nuclear magnetic moment; $P_{0}$ and $P_{n 0}$ are the thermal equilibrium values of the electron and nuclear polarizations; $\Delta=v_{e}-v ;$ and $\beta_{L}=1 / k T_{L}$, where $T_{L}$ is the lattice temperature. $W_{0}$ is the transition probability for an electron spin flip under influence of the microwave field; $W_{n}^{ \pm}$refer to the transition probabilities for a nuclear spin flip as a consequence of the forbidden transitions, while $W_{e}^{ \pm}$refer to the transition probabilities for a forbidden electron spin transition; they have maxima at $\Delta= \pm v_{n}$, respectively. The local internal magnetic field $H_{L}$ and the corresponding frequency $v_{L}$ are defined by the relation ${ }^{8,9}$

$$
\left(h v_{L}\right)^{2}=\left(g \mu_{\mathrm{B}} H_{L}\right)^{2}=\left[\operatorname{Tr}\left(H_{S S}^{0}\right)^{2}\right] / \operatorname{Tr} S_{z}^{2}
$$

where $g$ is the $g$ factor and $\mu_{\mathrm{B}}$ is the Bohr magneton. The time constants $\tau_{\mathrm{S} n}$ and $\tau_{n S}$ characterize, respectively, the relaxation of the spin-spin interaction reservoir to the nuclear Zeeman reservoirs and vice versa. The spin lattice relaxation times are denoted by $T_{1 n}, T_{1 e}$, and $T_{1 s s}$. There exist the following relations between the different parameters : ${ }^{6}$

$$
\frac{\tau_{S n}}{\tau_{n S}}=\frac{C_{S S}}{C_{\mathrm{Zn}}}=\frac{n_{e}}{n_{n}} \frac{v_{L}^{2}}{v_{n}^{2}} \frac{S(S+1)}{I(I+1)}
$$

and

$$
W_{e}^{ \pm}=\frac{n_{n}}{n_{e}} \frac{I(I+1)}{S(S+1)} W_{n}^{ \pm}=f W_{0}
$$

Here $C_{S S}$ and $C_{Z_{n}}$ are the heat capacities of the spin-spin interaction reservoir and the nuclear Zeeman reservoir, while $n_{e}$ and $n_{n}$ denote the number of electrons and nuclei in the sample, respectively. The "leakage" factor $f$ is defined as

$$
f=\sum_{k} 4\left|\varepsilon_{i k}\right|^{2}
$$

where $\varepsilon_{i k}$ is the dipolar coupling coefficient between an electron spin $i$ and a nuclear spin $k$ at a distance $r$ from the electron spin. ${ }^{1,2}$ The value of $4\left|\varepsilon_{i k}\right|^{2}$, 
averaged over all possible orientations of $\bar{r}$, is given by ${ }^{2}$

$$
4\left|\varepsilon_{i k}\right|^{2} \approx\left(\frac{\text { dipolar energy }}{\text { nuclear Zeeman energy }}\right)^{2} \approx \frac{3}{10}\left(\frac{g \mu_{\mathrm{B}}}{H r^{3}}\right)^{2}
$$

We assume that the electron spins all have the same nuclear environment; thus the summation in Eq. (12) is independent of $i$. If the nuclear spin lattice relaxation proceeds via the forbidden transitions with the electron spins, ${ }^{2,41}$ so that $T_{1 e} / T_{1 n}=W_{n}^{ \pm} / W_{0}$, then the average value of $\sum_{k} 4\left|\varepsilon_{i k}\right|^{2}$ is $n_{n} T_{1 e} / n_{e} T_{1 n}$, at least at low values of $P_{e}$, because at high electron polarizations, $T_{1 n}$ and consequently $f$ has to be multiplied by the factor $\left(1-P_{e} P_{0}\right){ }^{2}$ Note that $f$, as defined by Eq. (12), is independent of the temperature and is proportional to $H^{-2}$.

For a homogeneous spin system, $\tau_{n S}$ can be estimated from the expression $^{7}$

$$
\tau_{n S}^{-1} \approx \frac{n_{e}}{n_{n}} f T_{2 e}^{-1} \frac{\int_{0}^{\infty} g(v) g\left(v-v_{n}\right) d v}{g\left(v_{e}\right)}
$$

where $T_{2 e}$ is the transverse relaxation time of the electron spins. The abovementioned formulas are valid for homogeneous spin systems subjected to dipolar interactions. However, similar formulas hold for inhomogeneous spin systems, provided that cross-relaxation within the spin system is sufficiently fast to establish a thermal equilibrium within the spin system. ${ }^{4,6}$ The only difference comes from the increased heat capacity of the electron spin-spin interaction reservoir, which then includes the additional "nonZeeman" energy corresponding to the increased width of the Zeeman levels given by $\operatorname{Tr} \rho^{\prime} \sum_{i}\left(v_{i}-\bar{v}\right) S_{z}^{i}$. Here $v_{i}$ is the Larmor frequency of an electron spin $i$, and $\bar{v}$ is the averaged Larmor frequency, while $\rho^{\prime}$ is given by

$$
\rho^{\prime}=\left\{1-\alpha \bar{v} \sum_{i}^{n_{e}} S_{z}^{i}-\beta v_{n} \sum_{k}^{n_{n}} I_{z}^{k}-\gamma\left[H_{S S}^{0}+\sum_{i}^{n_{e}}\left(v_{i}-\bar{v}\right) S_{z}^{i}\right]\right\} / \operatorname{Tr} 1
$$

The symbol 1 denotes the unit matrix. If this is taken into account, Eq. (9) reads

$$
\frac{1}{2}\left(v_{L}^{2}+M_{2}\right) \dot{\gamma}=-W_{0}^{i} \Delta_{i}\left(P_{i}+\frac{1}{2} \gamma \Delta_{i}\right)-\frac{1}{2}\left(a v_{L}^{2}+M_{2}\right)\left[\left(\gamma-\beta_{L}\right) / T_{1 e}\right]
$$

where $\Delta_{i}=v_{i}-v$ and $M_{2}=\sum_{i}^{n_{e}}\left(v_{i}-\bar{v}\right)^{2}$ is the second moment of the inhomogeneous ESR line, normalized such that $\sum 1=1$.

In Eq. (16) we neglected terms corresponding to simultaneous spin flips of two or more spins, thus assuming that $f \ll 1$ and also $\tau_{S n}^{-1} \propto f T_{2 e}^{-1} \ll T_{1 e}^{-1}$. These conditions are likely to be fulfilled in a high magnetic field, since $f \propto H^{-2}$ and $T_{1 e}^{-1} \propto H^{5} / P_{e}$, if the direct process for electron spin lattice relaxation dominates. ${ }^{2,7}$ Furthermore, we replaced $T_{1 \text { sS }}^{-1}$ by $a / T_{1 e}$, where the 
constant $a$ equals 3 in many cases. ${ }^{7}$ Equation (16) appears to be a good approximation for some of the experiments described later.

The consequences of slightly off-resonance irradiation can be summarized as follows: The difference between the photon and Zeeman energies is absorbed by the spin-spin interaction reservoir, and the corresponding change in the temperature of this reservoir is transmitted to the nuclei via the forbidden microwave-induced and cross-relaxation transitions. This thermal coupling is represented in Eq. (8) by the terms $\left(W^{+}+W^{-}+\right.$ $\left.1 / \tau_{n S}\right)(\beta-\gamma)$. If the sum of these terms is large compared with spin lattice relaxation, then the nuclei may obtain a temperature equal to that of the spin-spin interaction reservoir. This condition will be fulfilled if $\left(S^{+}+S^{-}+\right.$ $\left.S^{\mathrm{cr}}\right) \gg 1$. We use the following definitions:

$$
S_{1} \equiv W_{0} T_{1 e}, \quad S^{ \pm} \equiv W^{ \pm} T_{1 n}, \quad S^{\mathrm{cr}} \equiv T_{1 n} / \tau_{n S}
$$

In this way the absolute value of the spin temperatures of the nuclear Zeeman reservoirs may be appreciably lowered, thus giving rise to dynamic polarization in the presence of a magnetic field. If the thermal contact with the spin-spin interaction reservoir is strong enough, the final polarizations of different nuclear spin species will thus correspond to equal spin temperatures $;{ }^{4}$ they all become polarized simultaneously in a microwave frequency range around $v_{e}$. The maximum nuclear polarization can be either larger or smaller than $\left|P_{e}\right|$ depending on the value of $v_{n}{ }^{4}$ Such behavior differs appreciably from that of dynamic polarization by means of the resolved solid-state effect; in that case, the optimum nuclear polarization may become equal to but not larger than $\left|P_{e}\right|$ and occurs at a microwave frequency which is different for different nuclear Larmor frequencies.

\subsection{Special Cases}

The steady-state solutions of the electron and nuclear polarizations can be obtained from Eqs. (7)-(9) by putting the left-hand sides to zero. However, such a solution is only valid at high temperatures. The extension of the spin temperature theory toward low temperatures causes difficulties, first because of the higher order terms in the spin temperatures, which make the expressions for the energy of a spin system more complicated. Furthermore, one may wonder if the thermodynamic model of two separated energy reservoirs, the Zeeman and the spin-spin interaction reservoirs, can still be applied at low temperatures, because at $T_{Z}$ equal zero all spins are in the lower energy state, so their relative spin orientation is fixed, which means $T_{S S}$ is fixed. ${ }^{42}$ Then one cannot use the concept of two separated energy reservoirs, because of the limited degrees of freedom of the spin-spin interaction reservoir. However, during irradiation the number of degrees of 
freedom of the spin-spin interaction reservoir quickly increases, and we will assume in the following that after irradiation the electron spin system can still be characterized by two Boltzmann distributions with temperatures $T_{\mathrm{Z}}$ and $T_{\mathrm{SS}}$, respectively, even if the initial polarization is close to one.

Unfortunately, under this latter condition the high-temperature approximation cannot be used to calculate the final spin temperature. However, a rather simple expression can still be obtained if the broadening of the Zeeman levels of the electron spin system is mainly inhomogeneous, for example, due to hyperfine interactions and/or $g$-factor anisotropy. Then the contribution of the dipolar energy to the energy of the spin-spin interaction reservoir can be neglected, which simplifies the expressions considerably. From conservation of energy and angular momentum and by making the above-mentioned assumption, Borghini ${ }^{4,28}$ obtained the following relation for the steady-state value of $\gamma=h / k T_{S S}$ :

$$
\sum_{i} \Delta_{i} P_{i}=\Delta P_{0}
$$

where

$$
\begin{gathered}
\Delta_{i}=v_{i}-v, \quad \Delta=\bar{v}-v, \quad \bar{v}=\sum_{i} v_{i}, \quad \sum_{i} 1=1, \\
P_{0}=\tanh \left(\frac{1}{2} \beta_{i} \bar{v}\right)
\end{gathered}
$$

Terms corresponding to the hyperfine structure of the paramagnetic centers were not written down, although they can easily be taken into account. ${ }^{28}$ The electron polarization $P_{i}$ can be written as

$$
P_{i}=\tanh \left(\frac{1}{2} \alpha v_{i}\right)=\tanh \left(\frac{1}{2} \gamma \Delta_{i}\right)
$$

Here we used Redfield's relation $\alpha v_{i}=\gamma \Delta_{i}$, which is only true for the steady state of a spin system after strong irradiation. ${ }^{43}$ Note that electrons with a different value of $\Delta_{i}$ also have different polarizations, in contrast to a homogeneous spin system, where all spins maintain an equal polarization during irradiation. $^{4}$

In case of anisotropic paramagnetic centers in disoriented solids, the summation in Eqs. (18a) and (18b) has to be done over all angles. Replacing the summation by integration, the left-hand side in Eq. (18a) becomes ${ }^{28}$

$$
\sum_{i} \Delta_{i} P_{i} \approx\left(2 \pi^{2}\right)^{-1} \int_{0}^{\pi} \int_{0}^{2 \pi} \Delta_{i} P_{i} \sin \theta d \theta d \phi
$$

where

$$
\Delta_{i}=\left(g_{i} \mu_{\mathrm{B}} H / h\right)-v
$$


The $g$-factor $g_{i}$ of an electron spin $i$ is given by

$$
g_{i}=\left(g_{1}^{2} \cos ^{2} \theta+g_{2}^{2} \sin ^{2} \theta \cos ^{2} \phi+g_{3}^{2} \sin ^{2} \theta \sin ^{2} \phi\right)^{1 / 2}
$$

The polar coordinates $\theta$ and $\phi$ define the direction of the external magnetic field with respect to the principal axis of the $g$-tensor, whose principal values $g_{1}, g_{2}$, and $g_{3}$ can be determined from electron spin resonance measurements. The steady-state value of $\gamma$ for a certain value of $\Delta P_{0}$ can be found by interpolation if the summation in Eq. (18a) is done for a sufficiently large range of the parameters $\gamma, \Delta$, and $P_{0}$. In 1,2-propanediol doped with paramagnetic $\mathrm{Cr}^{\mathrm{v}}$ complexes, there exists a strong thermal contact between the nuclei and the electron spin-spin interaction reservoir, especially during microwave irradiation. ${ }^{44}$ Therefore all nuclei will obtain a temperature equal to $\gamma$, and the polarizations of different nuclear spin species can be found from the Brillouin function.

The finite nuclear spin lattice relaxation causes a "leakage" of the polarization to the lattice. The nuclear relaxation rate in 1,2-propanediol doped with $\mathrm{Cr}^{\mathrm{V}}$ complexes was found to be proportional to the theoretical expression given by ${ }^{2}$

$$
T_{1 n}^{-1} \propto T_{1 e}^{-1} H^{-2} \sum_{i}\left(1-P_{i} P_{0}\right)
$$

except for values of $T_{1 n}$ longer than two days. ${ }^{23}$ Then the field and temperature dependence of $T_{1 n}$ appeared to be less steep compared to predictions based on Eq. (21).

The leakage can be taken into account by increasing the heat capacity of the electron spin-spin interaction reservoir with a term proportional to the heat capacity of the nuclear Zeeman reservoirs. At low temperatures this term, which has to be added to the left-hand side of Eq. (18a), becomes for nuclear spins one-half ${ }^{4}$

$$
\sum_{i} f v_{n} P_{n}\left(1-P_{0} P_{i}\right)
$$

The value of the leakage factor $f=n_{n} T_{1 e} / n_{e} T_{1 n}$ was estimated from the spin concentrations and measured spin lattice relaxation times. $T_{1 n}^{-1}$ is by definition the part of the inverse relaxation time that occurs via direct interaction with the lattice. This value of $T_{1 n}^{-1}$ does not need to correspond to the measured inverse relaxation time, since the relaxation may also occur via the electron spin-spin interaction reservoir. ${ }^{5-7,39}$ However, it is difficult to separate this contribution from the observed inverse relaxation time. We therefore used the values of the measured relaxation times, which thus give a lower limit to the calculated polarizations.

The $g$-factor anisotropy of paramagnetic $\mathrm{Cr}^{\mathrm{v}}$ complexes in propanediol is given in Section 3. The maximum ratio of $\gamma / \beta_{L}$ as well as the corresponding 


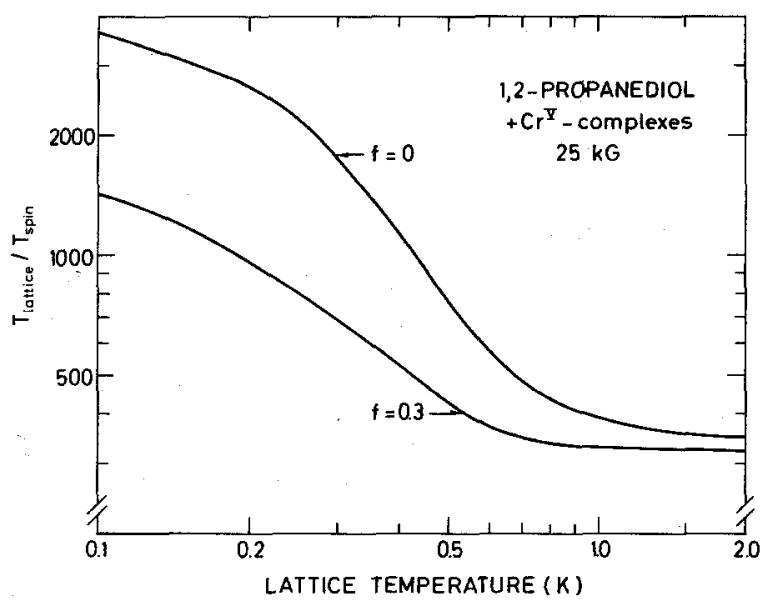

Fig. 2a. The ratio of the optimum inverse spin temperature and the inverse lattice temperature as a function of temperature in a $25 \mathrm{kG}$ magnetic field for two values of the leakage factor $f$. The curves were calculated from Eqs. (18), (20), and (22).

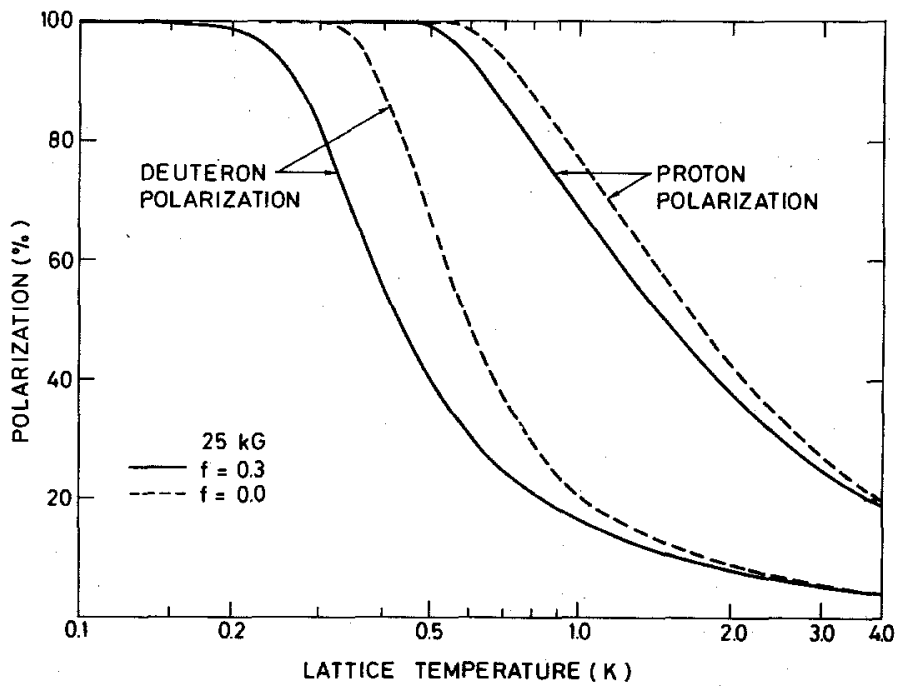

Fig. $2 b$. The maximum proton and deuteron polarizations in a $25 \mathrm{kG}$ magnetic field versus temperature as calculated from Eqs. (18), (20), and (22) and the Brillouin function. For the dashed curves the leakage term was taken to be zero; for the solid curves the leakage factor was estimated from the measured relaxation times. 




Fig. 3. The optimum proton polarization in 1,2-propanediol doped with $\mathrm{Cr}^{\mathrm{v}}$ complexes, as a function of magnetic field for three different temperatures. The leakage factor for each field value was estimated from the measured relaxation times and spin concentrations.

values of the proton and deuteron polarization were calculated from Eqs. (18a), (20a), and (22) for different magnetic field values and temperatures. Some results are presented in Figs. 2-4. The curves were optimized with respect to the microwave frequency. The value of $\Delta$ at which maximum polarizations were obtained varied slightly as a function of temperature. It changed, for example, from 160 to $200 \mathrm{MHz}$ at $25 \mathrm{kG}$ if the lattice temperature decreased from 1 to $0.4 \mathrm{~K}$, which is in good agreement with experimental results.

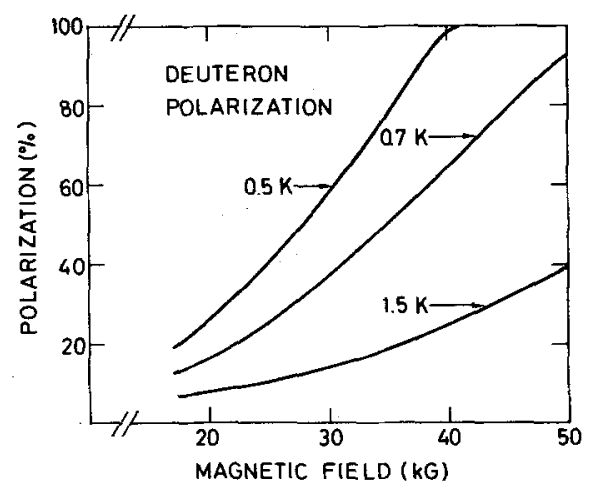

Fig. 4. As in Fig. 3, but now for the deuteron polarization. 
At low temperatures the enhancement starts to increase (see Fig. 2), which may seem rather surprising since the right-hand side of Eq. (18a) approaches a constant value when the polarization approaches one; then it could be expected that $\gamma$ also approaches a constant value, and thus $\gamma / \beta_{L}$ would decrease. However, it should be realized that the left-hand side of Eq. (18a) also shows a "saturation" behavior, so a small increase in $\Delta P_{0}$ may cause a large increase of $\gamma$. Of course, the integration of Eq. (20a) should be sufficiently precise to distinguish such small effects (a relative accuracy of $10^{-10}$ was needed at the lowest temperatures).

It is sometimes thought that the influence of the leakage factor will be negligible at low temperatures, because of the factor $\sum_{i}\left(1-P_{i} P_{0}\right)$. However, as can be seen from Fig. 2, the enhancement may be doubled at $0.5 \mathrm{~K}$ if $f$ varies from 0.3 to 0 , and at lower temperatures the difference starts to be even more important.

In the next section we will compare these curves with measurements which indicate both the increase of the enhancement at low temperatures and the important role of the leakage factor.

Previously we considered the limit of dynamic polarization at low temperatures under strong saturation conditions. There exists no exact theory for the case of weak saturation conditions at low temperatures, but by making a few simplifying assumptions, an estimate of the polarization as a function of the microwave frequency may be made.

The evolution of the electron polarization under microwave irradiation is given by Eq. (7), which is valid at low values of $P_{e}$. However, under the condition

$$
\frac{1}{2} \gamma \Delta \ll P_{e}
$$

which is true if the system is close to thermal equilibrium with the lattice, the term $\left(P_{e}+\frac{1}{2} \gamma \Delta\right)$ may be replaced by $P_{e}$, and Eq. (7) reduces to

$$
\dot{P}_{e}=-W_{0} P_{e}-\left(1 / T_{1 e}\right)\left(P_{e}-P_{0}\right)
$$

Here we assumed that terms proportional to $f$ are negligible. Equation (24) is just the classical one in case of negligible spin-spin interactions, which is valid without restrictions on the temperature. The condition (23) will only be fulfilled under weak saturation conditions. In that case the spin-spin interaction reservoir will hardly be cooled. Therefore we used the hightemperature approximation for the spin-spin interaction energy, assuming also that the spin lattice relaxation of this energy is exponential at low temperatures. Under these assumptions the steady-state solution of the proton polarization as a function of microwave frequency is easily obtained 
from Eqs. (8), (16), and (17):

$$
P(\mathrm{H})=\frac{S^{-}-S^{+}+\Delta v_{H} S_{1} S^{\mathrm{cr}} / \Omega^{2}}{1+S^{+}+S^{-}+S^{\mathrm{cr}}}
$$

Here $\Omega^{2}$ is defined as $a v_{L}^{2}+M_{2}$ and we replaced $P_{i}+\frac{1}{2} \gamma \Delta_{i}$ by $P_{i} \approx-1$. Terms proportional to $f, \beta_{L}$, and $\gamma\left(\Delta \pm v_{n}\right)$ were neglected. The dimensionless saturation parameters $S_{1}$ and $S^{ \pm}$, defined by Eq. (17), are assumed to have the same shape as the ESR line with maxima at $v_{e}$ and $v_{e} \pm v_{n}$, respectively, with $v_{n}=v_{\mathrm{H}}$ for proton spins. The values of these maxima are equal, as follows from the equality

$$
T_{1 e} / T_{1 n}=W_{n}^{ \pm} / W_{0}
$$

which arises from the fact that $T_{1 n}$ and $W_{n}^{ \pm}$are related to $T_{1 e}$ and $W_{0}$, respectively, via the dipolar coupling coefficient. The first two terms in the numerator of Eq. (25) can be interpreted as the solid-state effect, while the third term then corresponds to dynamic polarization arising from the thermal contact with the dynamically cooled electron spin-spin interaction reservoir.

Since the three terms have maxima at different microwave frequencies, they can be distinguished from each other if the ESR line is sufficiently narrow. ${ }^{4}$

If one uses a partially deuterated sample, the deuteron polarization will arise mainly from the thermal contact with the spin-spin interaction reservoir if the deuteron Larmor frequency $v_{\mathrm{D}}$ is small compared with the ESR linewidth, as is usually the case. Then the third term of the numerator in Eq. (25) will dominate, since

$$
S^{\mathrm{cr}} \gg S^{ \pm}
$$

as follows from a simple estimate of $S^{\text {cr }}$, using Eq. (14), and from the assumption of weak saturation $\left(S^{ \pm} \ll 1\right)$. A large value of $S^{\text {cr }}$ implies a strong thermal contact between the nuclei and the spin-spin interaction reservoir and they eventually reach an equal spin temperature. Assuming this to be true, the deuteron polarization can be written as

$$
P(\mathrm{D})=\frac{2}{3} \gamma v_{\mathrm{D}}=\frac{4}{3} \Delta v_{\mathrm{D}} S_{1} / \Omega^{2}
$$

In order to obtain the right-hand side of this equation, we assumed again $P_{i}+\frac{1}{2} \gamma \Delta_{i} \approx-1$ and $f=0$. From Eq. (16) the value of $\gamma$ is then found to be $2 \Delta S_{1} / \Omega^{2}$.

The expressions (25) and (28) will be compared with measurements of the polarization as a function of microwave frequency in experiments with the free radical BDPA, which exhibits such a narrow ESR line. ${ }^{28}$ 


\section{COMPARISON BETWEEN THEORY AND EXPERIMENTAL RESULTS}

\subsection{Results on $\mathrm{Cr}^{\mathrm{V}}$ Complexes}

We will first discuss the experimental results obtained in diols doped with paramagnetic $\mathrm{Cr}^{v}$ complexes. The first dynamic polarization experiments in ethanediol were performed by Fedotov on liquid samples. ${ }^{45}$ Later, high polarizations were obtained in frozen ethanediol at $25 \mathrm{kG}$ field, ${ }^{22}$ after which these measurements were extended to 1,2 -propanediol, ${ }^{23,46,47}$ partially deuterated ethanediol-D4, ${ }^{24}$ and 1,2-propanediol-D6. ${ }^{44}$ The observed high proton polarizations near $100 \%$ make these materials suitable for use in polarized targets. ${ }^{46,47}$ Most of the data are available for normal and partially deuterated 1,2-propanediol doped with about $10^{20} \mathrm{Cr}^{\mathrm{v}}$ complexes $/ \mathrm{cm}^{3}$, a concentration which has been found to give maximum polarization. ${ }^{47}$ The following results will refer to these two materials. Details about experimental techniques and sample preparation can be found elsewhere. ${ }^{24,44,47} \mathrm{The}^{\mathrm{Cr}} \mathrm{V}$ complex was discovered by Garif'yanov et al., ${ }^{48}$ who studied its ESR spectrum in ethanediol using a $3 \mathrm{kG}$ magnetic field. From the spectrum, they were able to deduce the most probable chemical structure of the complex and the fact of axial symmetry at the position of the $\mathrm{Cr}^{\mathrm{V}}$ ion. At $25 \mathrm{kG}$ the $\mathrm{g}$ factor anisotropy is the dominant broadening mechanism in a solid. ${ }^{49}$ The ESR spectrum of $\mathrm{Cr}^{\mathrm{v}}$ complexes in 1,2-propanediol, obtained at $0.5 \mathrm{~K}$ with a $4 \mathrm{~mm}$ transmission ESR spectrometer, is shown in Fig. 5. From this spectrum we deduced the $g$-factor anisotropy to be

$$
\frac{\Delta g}{g}=\frac{g_{\perp}-g_{\|}}{g} \cong 4 \times 10^{-3}
$$

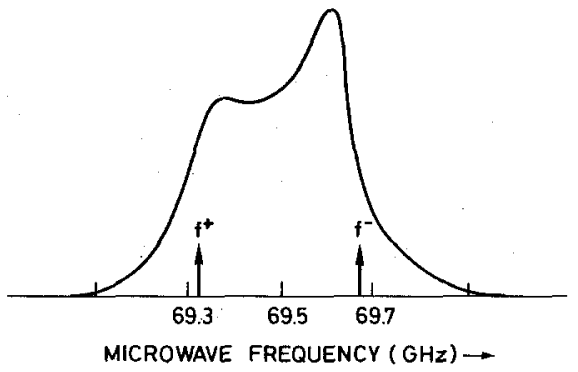

Fig. 5. The ESR absorption spectrum of propanediol with a $\mathrm{Cr}^{\mathrm{V}}$ concentration of $16 \times 10^{19}$ spins $/ \mathrm{cm}^{3}$ in a $25 \mathrm{kG}$ magnetic field at $0.5 \mathrm{~K}$. The arrows indicate the microwave frequency where optimum polarization is obtained $\left(f^{+}\right.$for positive and $f^{-}$for negative polarization). 
where $g=1.985$. The hyperfine interaction with the ${ }^{53} \mathrm{Cr}$ isotope $\mathrm{e}^{48}$ is much smaller than the splitting arising from the $g$-factor anisotropy, and since the natural abundance of ${ }^{53} \mathrm{Cr}$ is also low $(\sim 10 \%)$, the hyperfine interaction was neglected in the calculations. In order to be able to evaluate the leakage factor, we determined the electron spin lattice relaxation time by measuring the recovery time of the ESR signal after saturation. With a concentration of $16 \times 10^{19} \mathrm{spins} / \mathrm{cm}^{3}$ the spin lattice relaxation time was found to be $38 \pm 2$ msec at a temperature of $0.5 \mathrm{~K}$ in a $25 \mathrm{kG}$ magnetic field. In Fig. $6 \mathrm{a}$ we show the observed proton and deuteron polarizations in a $25 \mathrm{kG}$ magnetic field as a function of temperature together with curves derived from Eqs. (18a) and (22). The points on the upper curve were obtained in normal propanediol $^{23}$ and the points on the lower curve in propanediol-D6. ${ }^{44}$

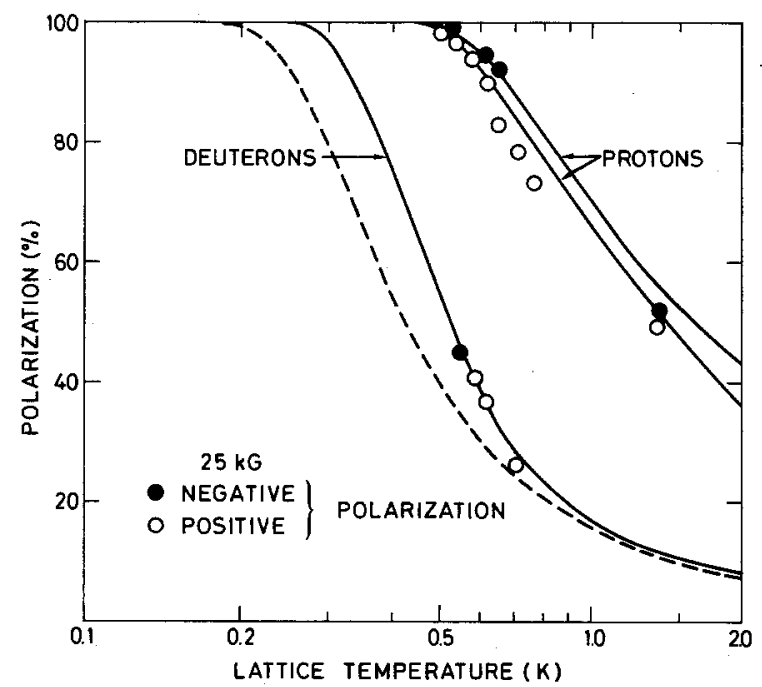

Fig. 6a. The temperature dependence of the proton and deuteron polarizations in 1,2-propanediol doped with $\mathrm{Cr}^{\mathrm{v}}$ complexes. The curves were calculated from Eqs. (18), (20), and (22) and the Brillouin function. The upper curve corresponds to the calculated negative proton polarizations, which are seen to be slightly higher than the positive polarizations. The leakage term was determined from the measured relaxation times; in the calculation of the deuteron polarization curve, the reduced heat capacity of the nuclear Zeeman reservoirs was taken into account. For comparison, the deuteron polarization is also shown if the reduced heat capacity for the deuterated sample was not taken into account (dashed line). The experimental points were obtained from Ref. 23 (upper curves) and Ref. 44 (lower curves). 


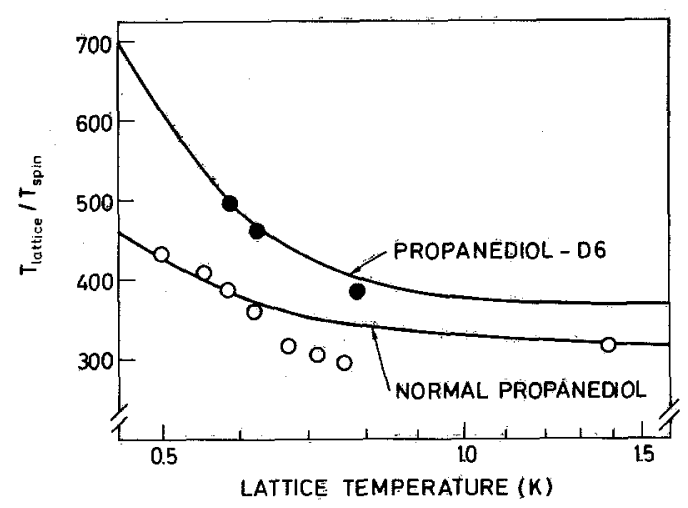

Fig. 6b. The "enhancement" of the inverse spin temperatures for normal and partially deuterated propanediol. The difference between the two samples arises from the smaller heat capacity of the nuclear Zeeman reservoirs in the deuterated sample. The curves were calculated from Eqs. (18), (20), and (22).

The temperature assigned to the experimental points cannot be determined easily, due to microwave heating of the sample. Fortunately, one thing can be measured rather precisely, namely the slope $\partial P / \partial T$, because at low temperatures the thermal contact between the sample and the He bath is mainly limited by the Kapitza boundary resistance. Then the rate of change of energy $\dot{Q}$ between the sample and the bath, which have temperatures $T_{s}$ and $T_{L}$, respectively, is given by

$$
\dot{Q} \propto\left(T_{s}^{4}-T_{L}^{4}\right)
$$

or

$$
T_{s}=C \dot{Q}^{1 / 4}
$$

at low temperatures. This means that $T_{s}$ is mainly determined by the microwave power, at least if $C$ is constant. This relation was verified by measuring the difference between $T_{s}$ and $T_{L}$ as a function of microwave power. ${ }^{23} \mathrm{By}$ using a microwave frequency far from paramagnetic resonance, the sample temperature could be determined accurately from the nuclear spin lattice relaxation time. The microwave power was determined by comparing it with the power of an electrical heater wrapped around the mixing chamber. We determined the maximum proton polarization for different microwave power levels: ${ }^{23}$ The sample temperature was calculated from Eq. (30b), using a value of $C$ equal to $2.9 \mathrm{~K} / \mathrm{W}^{1 / 4}$, which was found to give the best fit to the calculated polarizations. The sample temperature around $1 \mathrm{~K}$ was 
determined by using the Kapitza resistance coefficient given by Boyes et al. ${ }^{50}$ At the highest polarizations the microwave power was about $0.5 \mathrm{~mW} / \mathrm{g}$ and the temperature of the ${ }^{3} \mathrm{He}-{ }^{4} \mathrm{He}$ mixture in the dilution refrigerator was $0.2 \mathrm{~K}$. It should be noted that a different value of $C$ would merely shift the experimental points horizontally, thus retaining the steep temperature dependence of the polarization. The optimum polarizations were obtained at a microwave frequency for which $|\Delta| \approx 200 \mathrm{MHz}$, which is in good agreement with the calculated value of $\Delta$.

At a constant temperature the negative polarization was observed to be slightly higher than the positive one, as was expected from the calculations (the upper curve in Fig. 6a corresponds to the calculated negative polarizations). This difference is connected with the asymmetric ESR line. $^{28}$ It had already been observed earlier that the proton polarization was higher in deuterated butanol than in normal butanol under given conditions. ${ }^{51}$

For the calculations of the curve for the deuteron polarization we used a slightly lower value of $f$ corresponding to the reduced heat capacity of the nuclear Zeeman reservoirs in the partially deuterated sample as compared to a normal sample (about a factor of three lower). As was calculated before, a small difference in the leakage term should still give appreciable variations, even at low temperatures. In Fig. $6 \mathrm{~b}$ the calculated enhancement of the inverse spin temperatures is shown for a normal and a deuterated propanediol sample together with the experimental points. The expected increase of the enhancement and the difference between a normal and a deuterated sample are evident. The curves in Fig. 6 were calculated under the assumption of a strong thermal coupling between the nuclei and the electron spin-spin interaction reservoir. This coupling can be observed from the evolution of the spin temperatures of different nuclear spin systems, which are made unequal beforehand, for example, by rf saturation of one of the spin species. ${ }^{24}$ The different spin termperatures than evolve toward each other with a time constant which was found to be one order of magnitude smaller than the nuclear relaxation times, but which shows the same temperature and field dependences. If microwaves are applied, the thermal contact time between protons and deuterons in propanediol-B6 is only a few seconds in a $25 \mathrm{kG}$ magnetic field. ${ }^{44}$ These phenomena, which were observed before in similar materials, ${ }^{24}$ can only be understood by assuming a strong thermal coupling between the nuclei and the electron spin-spin interaction reservoir. As a consequence of this coupling, all nuclear spins (protons, deuterons, and ${ }^{13} \mathrm{C}$ nuclei) were found to have equal spin temperatures during polatization buildup as well as in the steady state, ${ }^{4.4}$ at least within the experimental errors of a few per cent. Such observations have been reported before for similar materials. ${ }^{24,52}$ 


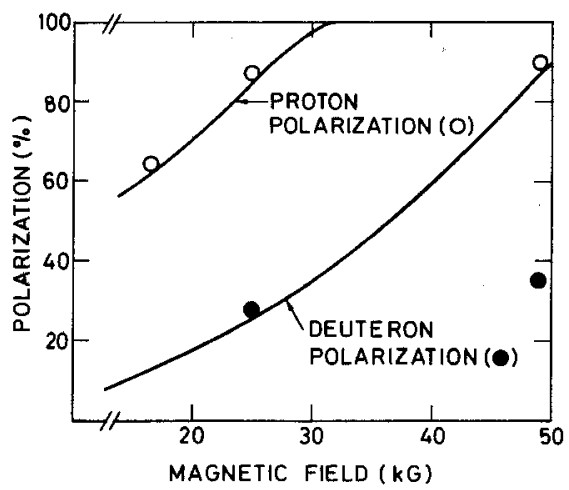

Fig. 7. The proton and deuteron polarizations in 1,2-propanediol doped with $\mathrm{Cr}^{\mathrm{v}}$ complexes at $0.7 \mathrm{~K}$ as a function of magnetic field. The polarization was measured at three field values $(17,25$, and $48 \mathrm{kG})$. The large deviation at $48 \mathrm{G}$ is discussed in the text.

Some measurements were done at different magnetic fields, ${ }^{53, *}$ namely at 48,25 , and $17 \mathrm{kG}$, at an estimated sample temperature of $0.7 \mathrm{~K}$. The points are shown in Fig. 7 together with the calculated curves. A rather large deviation is found at $48 \mathrm{kG}$. There may be several reasons for this: The electron spin system is not completely saturated because of the short electron spin lattice relaxation time (about $1 \mathrm{msec}$, if we extrapolate the value at $25 \mathrm{kG}$ ), ${ }^{44}$ or the thermal contact of the nuclei with the electron-spin interaction reservoir becomes too poor. The latter may be due to a slow cross-relaxation within the electron spin system, since the $g$-factor anisotropy will cause an appreciable inhomogeneous broadening at $50 \mathrm{kG}$. Also, the factor $\sum_{i}\left(1-P_{i} P_{0}\right)$ then becomes very small. This factor arises simply from the fact that at high polarizations there are hardly any electrons with spin up, thus reducing the cross-relaxation rate, since a combination of spin up and spin down is a necessary condition for a flip-flop transition. ${ }^{54} \mathrm{By}$ using a higher microwave power at $48 \mathrm{kG}(6 \mathrm{~mW} / \mathrm{g}$ instead of $1 \mathrm{~mW} / \mathrm{g}$ at $25 \mathrm{kG})$, we achieved a proton polarization of about $90 \%$, but the sample temperature was then about $0.9 \mathrm{~K}$, and the use of a ${ }^{3} \mathrm{He}$ evaporation cryostat had little advantage over a ${ }^{4} \mathrm{He}$ cryostat, in which similar polarizations have been observed at $50 \mathrm{kG} .{ }^{55}$

\subsection{Results on the Free-Radical BDPA}

Several dynamic polarization experiments have been performed with the free radical BDPA., ${ }^{41,25}$ This radical shows a resonance absorption line-

*The author is indebted to Dr. S. Mango for the measurements at $17 \mathrm{kG}$, which were performed at SIN, Zürich. 
Fig. 8. ESR signal of toluol doped with the free radical BDPA $\left(5 \times 10^{19}\right.$ spins per $\left.\mathrm{cm}^{3}\right)$ in a $25 \mathrm{kG}$ magnetic field at $0.5 \mathrm{~K}$.

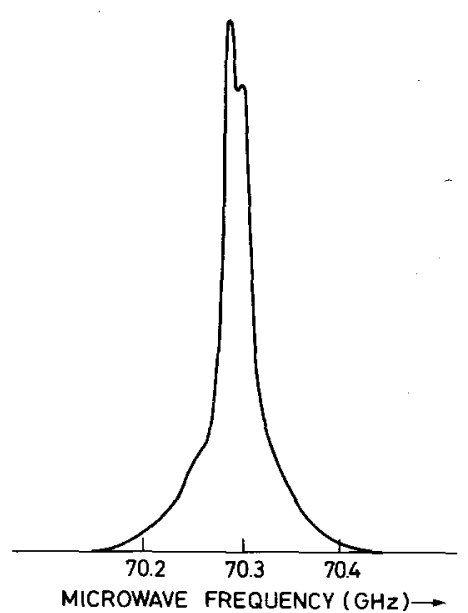

width of only $20 \mathrm{MHz}$ at $0.5 \mathrm{~K}$ in a $25 \mathrm{kG}$ magnetic field (see Fig. 8). This width is about the same as the one in a frozen sample at $3 \mathrm{kG}$ and a temperature of about $-100^{\circ} \mathrm{C}$; thus in a high magnetic field the $g$-factor anisotropy is not the dominant broadening mechanism.

Since the ESR linewidth is rather small as compared to the proton Larmor frequency in a $25 \mathrm{kG}$ magnetic field $(\sim 106 \mathrm{MHz})$, one expects for protons a resolved solid-state effect, eventually accompanied by dynamic polarization by cooling of the electron spin-spin interaction reservoir. ${ }^{4,25}$ Only this latter mechanism is expected for deuterons and ${ }^{13} \mathrm{C}$ nuclei, because their Larmor frequencies are of the same order as the ESR linewidth, and cross-relaxation within the narrow ESR line will be fast compared with the electron spin lattice relaxation time, which was measured to be $1.6 \pm 0.2$ msec in a toluol sample doped with $5 \times 10^{19}$ spins $/ \mathrm{cm}^{3}$ at a temperature of $0.5 \mathrm{~K}$ in a $25 \mathrm{kG}$ magnetic field. ${ }^{44}$ Since the nuclear spin lattice relaxation times were measured to be at least several minutes under the experimental conditions, the leakage factor is smaller than $10^{-2}$ and we therefore neglect it.

The polarizations of protons and deuterons as a function of the microwave frequency in partially deuterated $m$-xylene (2,2-D6) doped with BDPA $\left(6 \times 10^{18}\right.$ spins per $\left.\mathrm{cm}^{3}\right)$ are shown in Figs. 9 and 10 . The experimental points were obtained from Ref. 25 . The way in which the curves were calculated will be discussed below. The large peaks of the proton polarization, occurring at frequencies for which $\Delta= \pm v_{\mathrm{H}}$, correspond to the resolved solid-state effect, while the inner ones are attributed to a thermal contact with the electron spin-spin interaction reservoir. Two small peaks were 


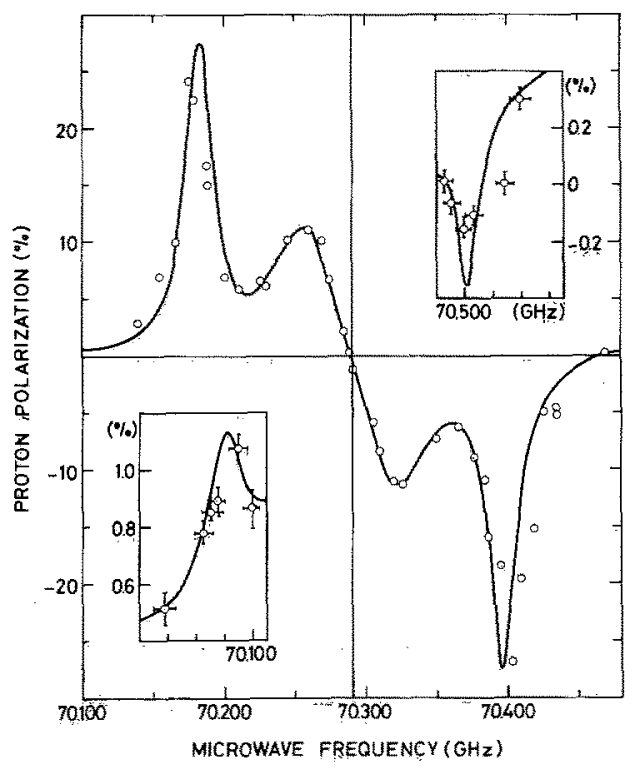

Fig. 9. Proton polarization versus microwave frequency in $m$-xylene-D6 doped with BDPA $\left(6 \times 10^{18}\right.$ spins $/ \mathrm{cm}^{3}$ ). The curve was calculated from Eq. (31). The insets show the double solid-state effect on an enlarged scale.

observed at microwave frequencies around $v=v_{e} \pm 2 v_{\mathrm{H}}$. These are due to a "double" solid-state effect in which two proton spins flip simultaneously with an electron spin in the opposite direction. The curve in Fig. 9 was calculated from the following formula :

$$
P(\mathrm{H})=\frac{\left(S^{-}+s^{-}\right)-\left(S^{+}+s^{+}\right)+\Delta v_{\mathrm{H}} S_{1} S^{\mathrm{cr}} / \Omega^{2}}{1+S^{+}+S^{-}+S^{\mathrm{cr}}}
$$

The terms $s^{ \pm}$, which are much smaller than $S^{ \pm}$, were added to Eq. (25) to take the "double" solid-state effect into account.

In this calculation the ESR line shape $g(v)$ was approximated by a Lorentzian with a half-width of $10 \mathrm{MHz}$. The $S^{+}$and $S^{-}$with maxima at $v_{e}+v_{\mathbf{H}}$ and $v_{e}-v_{\mathbf{H}}$ were taken to be proportional to $g(v)$. Their absolute values were obtained by determining the maximum values of $S^{ \pm}$from the measured polarization time $\tau_{ \pm}$at the solid $=$state effect frequencies, using the expression $^{2}$

$$
\tau_{ \pm}=T_{1 \mathrm{H}}\left[1 /\left(S^{ \pm}+1\right)\right]
$$




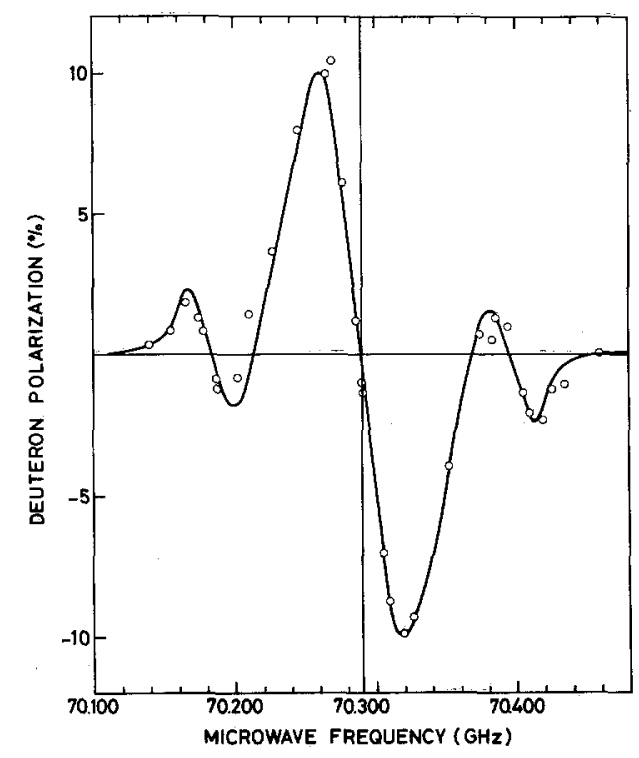

Fig. 10. Deuteron polarization versus microwave frequency in $m$-xylene=D6 doped with BDPA $\left(6 \times 10^{18}\right.$ spins $\left./ \mathrm{cm}^{3}\right)$. The curve was calculated from Eq. (34).

The polarization time was measured to be $20 \mathrm{~min}$ with a microwave power of $7 \mathrm{~mW} / \mathrm{g}$, while $T_{1 \mathrm{H}}$ was $30 \mathrm{~min}$ at the estimated sample temperature of $0.75 \mathrm{~K}$, yielding a maximum value of $S^{ \pm}=0.5$.

The determination of $S_{1}$ as a function of microwave frequency is difficult, since the average microwave field strength in the cavity will decrease if the frequency is near paramagnetic resonance. This effect may be appreciable because of the high filling factor of the cavity $(\sim 0.5)$ and the high concentration of paramagnetic centers. The variation of the microwave field strength was measured as a function of microwave frequency with a bolometer, consisting of a carbon resistor which was placed inside the multimode cavity opposite the entrance of the waveguide; this had a resolution better than 0.5 $\mathrm{dB}$ at low temperature. ${ }^{23}$ The change in microwave field strength made $S_{1}$ a slightly broader function than $g(v)$. It could be approximated rather well by a Gaussian function with a half-width of $35 \mathrm{MHz}$.

Absolute values of the ratio $S_{1} / \Omega^{2}$ were obtained by fitting its value to the measured deuteron polarization, since in that case there are no other unknown quantities for microwave frequencies around $v_{e}$. This yielded

$$
S_{1} / \Omega^{2}=\left(3 \times 10^{-4}\right) \exp \left(-\Delta^{2} / 1800\right)
$$

where the parameters are expressed in $\mathrm{MHz}$. 
Since the value of $\Omega^{2}$ is of the order of $100 \mathrm{MHz}^{2}$, the value of $S_{1}$ is rather low compared with $S^{ \pm}$. This should not be surprising, since at low temperatures $T_{1 \mathrm{H}} \propto T_{1 e} F\left(P_{e}\right)$; thus $S^{ \pm}=S_{1} F\left(P_{e}\right)$, where $F\left(P_{e}\right)=1 /\left(1-P_{0} P_{e}\right)$ may vary between 10 and 20 , depending on the microwave frequency. Such a low value of $S_{1}$ justifies the use of Eqs. (25) and (28), which were derived under the assumption of weak saturation.

The value of $S^{\text {cr }}$ was taken to be 0.25 in order to fit the experimental points of $P(\mathrm{H})$ at the inner peaks. This rather low value expresses that the thermal contact of the proton spin system with the lattice is four times stronger than the contact with the electron spin-spin interaction reservoir. This is not surprising, since the proton Larmor frequency $v_{\mathrm{H}}$ is large compared with the ESR linewidth. Therefore it will be difficult to find two electron spins with a frequency difference of the order of $v_{H}$, which are at the same time sufficiently close in space to perform a flip-flop transition.

The saturation parameters $s^{ \pm}$of the double solid-state effect were taken proportional to $g(v)$ with maxima at $v_{e} \pm 2 v_{\mathbf{H}}$ equal to 0.007 , which is two orders of magnitude lower than $S^{ \pm}$.

The deuteron polarization as a function of microwave frequency shows four additional peaks at frequencies for which $|\Delta|=v_{\mathrm{H}} \pm v_{\mathrm{D}}$, besides the two expected peaks at frequencies around $v_{e}$. They are attributed to a double solid-state effect, in which one electron, one proton, and one deuteron spinflip simultaneously.

The curve drawn there was calculated from Eq. (28), in which four terms corresponding to this double solid-state effect were added:

$$
P(\mathrm{D})=+\frac{4}{3} \Delta v_{\mathrm{D}} S_{1} / \mathbf{\Omega}^{2}+S_{\mathrm{D}_{1}}^{-}-S_{\mathrm{D}_{1}}^{+}+S_{\mathrm{D}_{2}}^{-}-S_{\mathrm{D}_{2}}^{+}
$$

The saturation parameters $S_{\mathrm{D}_{1}}^{ \pm}$and $S_{\mathrm{D}_{2}}^{ \pm}$were taken proportional to $g(\Delta)$ with a maximum value of 0.03 at $v_{e}-v_{\mathrm{H}} \pm v_{\mathrm{D}}$ and $v_{e}+v_{\mathrm{H}} \pm v_{\mathrm{D}}$, respectively. Of course the double solid-state effect depends also on the proton polarization, but since this is small compared with the electron polarization, $S_{\mathrm{D}_{1}}$ and $S_{\mathrm{D}_{2}}$ were simply taken proportional to $P_{e} \approx-1$.

The ratio $S_{\mathrm{D}_{1}}^{ \pm} / s^{ \pm}$is about 4 . This order of magnitude was expected from the factor $I(I+1)$ in the expressions for the transition probabilities [see Eq. (11)]. This factor is about three times larger for deuterons than for protons.

By using Eq. (34), it was assumed that for deuterons the thermal contact with the electron spin-spin interactions occurs mainly via cross-relaxation transitions, which are proportional to $\int_{0}^{\infty} g(v) g\left(v-v_{n}\right) d v$ for a homogeneous ESR line [see Eq. (14)]. By using the value of $S^{\mathrm{cr}}$ for protons $(\sim 0.25)$, one can estimate the corresponding factor for deuterons to be 20 , which is indeed much larger than the saturation parameters for the forbidden transitions, so that condition (27) will be fulfilled. 


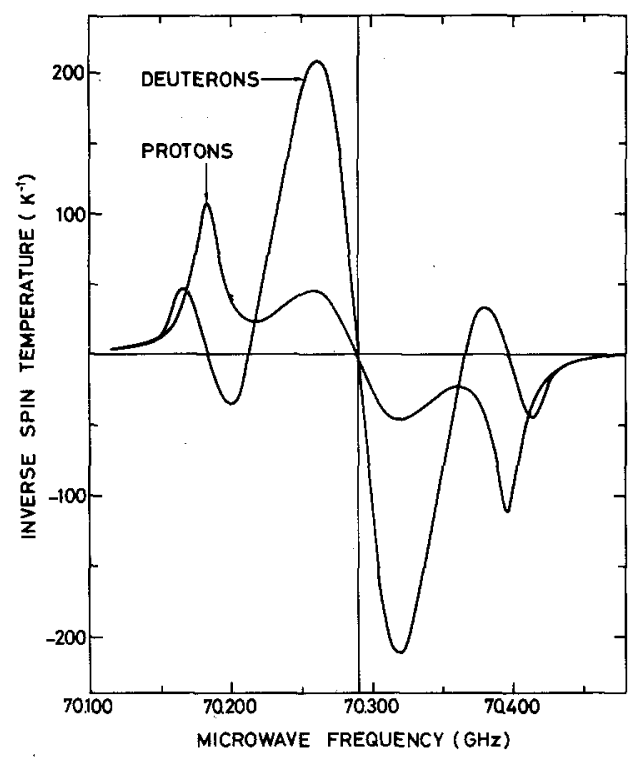

Fig. 11. The inverse spin temperatures of protons and deuterons in $m$-xylene doped with BDPA as calculated from Eqs. (31) and (34)-(36).

It is interesting to compare the spin temperatures of protons and deuterons. As mentioned before, these become equal in the case of a strong thermal contact with the electron spin-spin interaction reservoir. Figure 11 shows the inverse spin temperatures of protons and deuterons as a function of microwave frequency as calculated from Eqs. (31) and (34) and using the relations

$$
\begin{aligned}
& T(\mathrm{H})^{-1}=2 k P(\mathrm{H}) / h v_{\mathrm{H}} \\
& T(\mathrm{D})^{-1}=3 k P(\mathrm{D}) / 2 h v_{\mathrm{D}}
\end{aligned}
$$

It can be seen that the deuteron spin temperature is about five times lower than the proton spin temperature at frequences around $v_{e}$; this is attributed to the poor thermal contact of the protons with the electron spin-spin interaction reservoir. ${ }^{25}$

\section{CONCLUSIONS}

In previous sections we have compared the spin temperature theory with dynamic polarization experiments performed in organic materials at low temperatures and in high magnetic fields. 
The application of the spin temperature theory to situations where the polarization of the spin system is close to one is not obviously valid, due to the limited number of degrees of freedom of the spin-spin interaction reservoir. ${ }^{42}$ However, this number increases rapidly during irradiation, and it appears that the concept of a spin-spin interaction reservoir is still useful in the experiments, as discussed before.

The order of magnitude of the calculated maximum polarization values of protons, deuterons, and ${ }^{13} \mathrm{C}$ nuclei in 1,2-propanediol doped with $\mathrm{Cr}^{\mathrm{V}}$ complexes, as calculated from Borghini's spin temperature model, is in good agreement with the observed values. An exact comparison between theory and experiment is difficult because of the uncertainty in the sample temperature during microwave irradiation. However, the relative values of the polarizations of different nuclei agree within a few percent with the ratios expected from the "equal spin temperature hypothesis," which is furthermore justified by the observed strong thermal coupling between the different nuclear spin species. Also, several other features, such as a steep temperature dependence of the polarizations and an increase of the enhancement factor for spin temperatures below $2 \mathrm{mK}$, are quite well reproduced by the measurements, thus leaving little doubt that the observed high polarizations are mainly due to thermal contact with the dynamically cooled electron spinspin interaction reservoir. This is the first time that a quantitative agreement between experimental results and the above-mentioned spin temperature model has been obtained.

Different mechanisms of dynamic polarization may act simultaneously. This was especially clear from experiments with the free radical BDPA. ${ }^{25}$ By making a few simplifying assumptions, the steady-state solutions of the proton and deuteron polarizations as a function of microwave frequency were obtained from the basic set of Eqs. (7)-(9). The solutions were at least qualitatively in agreement with the measurements, which showed clearly the different features of each term in these equations. These features can be summarized as follows: Saturation of the forbidden transitions causes dynamic polarization by the solid-state effect or double solid-state effect, and the thermal contact with the electron spin-spin interaction reservoir causes polarization if this reservoir is cooled by off-resonance saturation of the allowed transitions.

\section{ACKNOWLEDGMENTS}

I wish to express my sincere thanks to the members of the Polarized Target group at CERN, whose help and experience in the field of dynamic polarization were indispensable during the course of this work. I especially wish to thank Drs. M. Borghini, K. Morimoto, T. O. Niinikoski, and F. Udo 
for many stimulating discussions and for their contributions during various stages of the experiments. Furthermore, I àm indebted to Prof. B. S. Blaisse, University of Delft, for a critical reading of the initial version of the manuscript.

\section{REFERENCES}

1. A. Abragam, Principles of Nuclear Magnetism (Oxford Univ. Press, London, 1961).

2. C. D. Jeffries, Dynamic Nuclear Orientation (Interscience Publishers, New York, 1963).

3. M. Goldman, Spin Temperature and Magnetic Resonance in Solids (Oxford Univ. Press, London, 1970).

4. M. Borghini, G. Shapiro, ed., in Proc. 2nd Internat. Conf. on Polarized Targets, Berkeley, 1971 (LBL 500, UC-34 Physics, National Technical Information Serviee, Springfield, Virginia, 1972), p. 1, and references quoted therein.

5. L. L. Buishvili, Zh. Eksp. Teor. Fiz. 49, 1968 (1965) [English transl., Soviet Phys.-JETP 22, 1277 (1966)]; L. L. Buishvili, M. D. Zviadadze, and G. R. Khutsishvili, Zh. Eksp. Teor. Fiz. 54, 876 (1968) [English transl., Soviet Phys.-JETP 27, 469 (1968)].

6. V. A. Atsarkin and M. I. Rodak, Uspekhi Fiz. Nauk 107, 3 (1972) [English transl., Soviét Phys.-Uspekhi 15, 251 (1973)], and references quoted therein.

7. W. Th. Wenckebach and N. J. Poulis, in 17th Congress Ampère, Turku, Finland, 1972 V: Hovi, ed. (North-Holland, Amsterdam, 1973), p. 120; W. Th. Wenekebach, T. J. B. Swanenburg, and N. J. Poulis, Phys. Reports, to be published.

8. A. Abragam and M. Borghini, in Progress in Low Temperature Physics, C. J. Corter, ed. (North-Holland Publishing, Amsterdam, 1964), Vol. IV, Chapter 8.

9. C. E. Byvik and D. S. Wollan, The Spin Temperature Theory of Dynamic Nuclear Polarization (NASA TR R-427, National Technical Information Service, Springfield, Virginia, 1974); D. S. Wollain, "Dynamic nuclear polarization with an inhomogeneous broadened ESR line," submitted to Phys. Rev. B.

10. La Direction de la Physique, CEN (eds.), Proc. Ist Internat. Conf. on Polarized Targets and Ion Sources, Saclay, 1966); (La Documentation Française, Saclay, 1966); G. Shapiro (ed.), Proc. 2nd Internat. Conf. on Polarized Targets, Berkeley, 1971 (LBL 500, UC-34 Physics, National Technical Information Service, Springfield, Virginia, 1972).

11. A. Kastler, C. R. Acad. Sci. B 233, 1444 (1951).

12. A. W. Overhauser, Phys. Rev. 89, 689 (1953); 92, 411 (1953).

13. J: Korringa, Phys. Rev. 94, 1388 (1954).

14. H. G. Beljers, L. van der Kint, and J. S. van Wieringen, Phys. Rev. 95, 1683 (1954):

15. A. Abragam, Phys. Rev: 98, 1729 (1955).

16. C. D. Jeffries, Phys. Rev. 106, 164 (1957).

17. N. Bloembergen and P. P. Sorokin, Phys. Rev. 110, 865 (1958).

18. A. Abragam and W. G. Proctor, C. R. Acad. Sci. B 246, 2253 (1958).

19. B. N. Provotorov, Zh. Eksp. Teor. Fiz. 41, 1582 (1961); [English transl., Soviet Phys.JETP 14, 1126 (1962)]; Fiz. Tverd. Tela 4, 2940 (1962); [English transl., Soviet Phys.-Solid State 4, 2155 (1963)]; Phys. Rev. 128, 75 (1962).

20. B. N. Provotorov, Zh. Eksp. Teor. Fiz. 42, 882 (1962); [English transl., Soviet Phys.-JETP 15, 611 (1962)].

21. M. Borghini, S. Mango, O. Runolfsson, and J. Vermeulen, in Proc. Ist Internat. Conf. on Polarized Targets and Ion Sources, Saclay, 1966, La Direction de la Physique, CEN, eds. (La Documentation Française, Saclay, 1966), p. 387.

22. H. Glättli, M. Odehnal, J. Ezratty, A. Malinovski, and A. Abragam, Phys. Lett. 29A, 5 (1969); A. Masaike, H. Glättli, J. Ezratty, and A. Malinovski, Phys. Lett. 30A, 63 (1969).

23. W. de Boer and T. O. Niinikoski, Nucl. Instr. Meth. 114, 495 (1974).

24. W. de Boer, M. Borghini, K. Morimoto, T. O. Niinikoski, and F. Udo, J. Low Temp. Phys. 15,249 (1974). 
25. M. Borghini, W. de Boer, and K. Morimoto, Phys. Lett. 48A, 244 (1974).

26. M. Goldman, M. Chapellier, Vu Hoang Chau, and A. Abragam, Phys. Rev. B 10, 226 (1974).

27. M. A. Kozhushner, Zh. Eksp. Teor. Fiz. 56, 246 (1969); [English transl., Soviet Phys.-JETP 29, $136(1969)]$.

28. M. Borghini, Phys. Rev. Lett. 20, 419 (1968).

29. T. R. Carver and C. P. Slichter, Phys. Rev. 92, 212 (1953).

30. M. Abraham, R. W. Kedzie, and C. D. Jeffries, Phys. Rev. 106, 165 (1957).

31. F. M. Pipkin and J. W. Culvahouse, Phys. Rev. 106, 1102 (1957).

32. C. D. Jeffries, Phys. Rev. 117, 1056 (1960).

33. E. Erb, J. L. Motchane, and J. Uebersfeld, C. R. Acad. Sci. B 246, 2121, 3050 (1958).

34. A. Abragam, J. Combrisson, and I. Solomon, C. R. Acad. Sci. B 247, 2237 (1958).

35. M. Abraham, M. A. H. McCausland, and F. N. H. Robinson, Phys. Rev. Lett. 2, 449 (1959).

36. C. F. Hwang and D. A. Hill, Phys. Rev. Lett. 19, 1011 (1967).

37. N. Bloembergen, E. M. Purcell, and R. V. Pound, Phys. Rev. 73, 679 (1948).

38. A. M. Portis, Phys. Rev. 91, 1070 (1953); 104, 584 (1956).

39. M. Borghini, Phys. Lett. 26A, 242 (1968).

40. M. A. Kozhushner and B. N. Provotorov, Fiz. Tverd. Tela 6, 1152 (1964); [English transl., Soviet Phys.,-Solid State 6, 1152 (1964)].

41. N. Bloembergen, Physica 15, 386 (1949); G. R. Khutsishvili, in 17th Congress Ampere, Turku, Finland, 1972, V. Hovi, ed. (North-Holland, Amsterdam, 1973), p. 35.

42. J. Philippot, Phys. Rev. 133, A471 (1964).

43. A. G. Redfield, Phys. Rev. 98, 1787 (1955); Science 164, 1015 (1969).

44. W. de Boer, Thesis (1974), University of Technology, Delft, The Netherlands [CERN 74-11 (1974)].

45. V. N. Fedotov, Zh. Eksp. Teor. Fiz. 53, 1982 (1967); [English transl., Soviet Phys.--JETP 26, 1123 (1968)].

46. W. Gorn and P. Robrish, in Proc. 2nd Internat. Conf. on Polarized Targets, Berkeley, 1971, G. Shapiro, ed. (LBL 500, UC-34 Physics, National Technical Information Service, Springfield, Virginia, 1972), p. 305.

47. W. de Boer, Nucl. Instr. Meth. 107, 99 (1973).

48. N. S. Garifyanov, B. M. Kozyrev, and V. N. Fedotov, Dokl. Akad. Nauk SSSR 178, 808 (1968); [English transl., Soviet Phys. Dokl. 13, 107 (1968)].

49. H. Glättli, in Proc. 2nd Internat. Conf. on Polarized Targets, Berkeley, 1971, G. Shapiro, ed. (LBL 500, UC-34 Physics, National Technical Information Service, Springfield, Virginia, 1972), p. 281.

50. E. Boyes, G. R. Court, B. Craven, R. Gamet, and P. J. Hayman, in Proc. 2nd Internat. Conf. on Polarized Targets, Berkeley, 1971, G. Shapiro, ed. (LBL 500, UC-34 Physics, National Technical Information Service, Springfield, Virginia, 1972), p. 403.

51. M. Borghini and K. Scheffler, Nucl. Instr. Meth. 95, 93 (1971).

52. M. Borghini and K. Scheffier, Phys. Rev. Lett. 26, 1362 (1971); M. Borghini and F. Udo, Phys. Lett. 43A, 93 (1973).

53. W. de Boer, M. Borghini, M. Rieubland, and F. Udo, "Dynamic polarization at ${ }^{3} \mathrm{He}$ temperatures in a $50 \mathrm{kG}$ magnetic field," unpublished results.

54. A. Abragam, in Proc. Ist Internat. Conf. on Polarized Targets and Ion Sources, Saclay, 1966, La Direction de la Physique, CEN, eds. (La Documentation Française, Saclay, 1966), p. 27.

55. M. Odehnal and V. Bouffard, Phys. Lett. 32A, 407 (1970). 